\title{
Stability of Converging Cylindrical Shock Waves*
}

\author{
Masato WATANABE** and Kazuyoshi TAKAYAMA**
}

\begin{abstract}
An experimental and numerical study was conducted on converging cylindrical shock waves. The goal of the present study was to clarify the nature of movement and instability in converging cylindrical shock waves. Experiments were conducted in an annular shock tube of $230 \mathrm{~mm}$ outer diameter and $210 \mathrm{~mm}$ inner diameter, connected to a cylindrical test section of $210 \mathrm{~mm}$ diameter. Double-exposure holographic interferometry was used to visualize the converging cylindrical shock waves. Incident shock Mach numbers ranged from 1.1 to 2.0 in air. A numerical simulation was conducted using the total variation diminishing (TVD) finite difference scheme. It was found in the experiments that the shock wave configuration was initially cylindrical, but gradually deformed with propagation towards the center and finally showed mode-four instability. This is attributable to the existence of initial disturbances, which were introduced by the struts supporting the inner tube of the annular shock tube. This trend was significant for stronger shock waves, indicating that at the last stage of shock wave convergence each initial perturbation of the converging cylindrical shock wave was amplified to form the triple point of a Mach reflection. Numerical results correctly predicted the experimental trend.
\end{abstract}

Key Words: Shock Wave, Stability, Cylindrical Convergence, Mach Reflection, Holographic Interferometry, TVD Finite Difference Scheme

\section{Introduction}

The stability of converging cylindrical shock waves is one of the unsolved problems of shock wave dynamics associated with shock wave focusing. Converging cylindrical and spherical shock waves are known to produce high temperatures and pressures at the center of convergence. Recently, shock wave focusing has been used for various interesting scientific and engineering applications ${ }^{(1)}$.

The first analytical work concerning converging shock waves was that of Guderley ${ }^{(2)}$, who obtained a self-similar solution for the convergence of cylindrical and spherical shock waves. Experimentally, Perry and Kantrowit $z^{(3)}$ produced converging cylindrical shock waves using a unique annular shock tube having an axisymmetric teardrop-shaped inner core. Since then, many studies of this problem have been conducted, experimentally ${ }^{(4),(5)}$, theoretically ${ }^{(6)}$ and

* Received 2nd September, 1991. Paper No. 90-0562 A

** Shock Wave Research Center, Institute of Fluid Science, Tohoku University, Katahira 2-1-1, Aoba, Sendai 980, Japan numerically ${ }^{(7) \sim(9)}$. However, the physics of converging shock waves and particularly the initiation of instability of converging shock waves have not yet been clarified, since this behavior is primarily dependent on nonlinear characteristics of shock waves.

In this investigation reported in this paper, converging cylindrical shock waves were generated using a coaxial annular shock tube which had an annular structure, similar to that of Perry and Kantrowit $z^{(3)}$. The coaxial annular shock tube enabled the transformation from a planar shock wave to a converging cylindrical shock wave to occur. The entire sequence of shock convergence was successfully visualized with double-exposure holographic interferometry. To interpret the experimental results, a numerical simulation, using the total variation diminishing (TVD) finite difference scheme $e^{(10),(11)}$, was conducted and good agreement was obtained between the experiment and the numerical simulation. Based on the numerical results, the relationship between the intensity of the initial disturbance and shock wave distortion was discussed in some detail. 


\section{Modeling of a Converging Cylindrical Shock Wave}

Figure 1 illustrates the process of conversion of a planar shock wave into a converging cylindrical shock wave. The planar shock wave in the shock tube was at first diffracted by a conical section to form a coaxial ring-shaped shock wave. This shape may suffer some distortion when passing over support struts, but subsequently returns to planar from after attenuation of the distortions. The coaxial ring-shaped shock wave thus formed was then rotated $90^{\circ}$ to be converted into a cylindrical shock wave moving towards the center.

\section{Numerical Simulation}

The TVD finite difference scheme ${ }^{(10),(11)}$ was applied to the two-dimensional unsteady Euler equations. Two computational models were considered, as shown in Fig. 2(a).

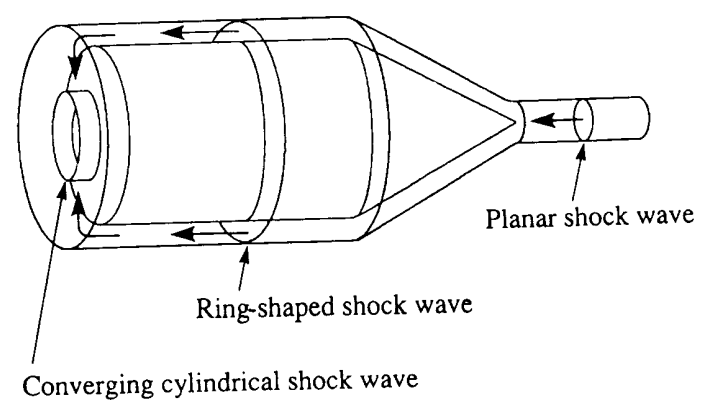

Fig. 1 Modeling of converging cylindrical shock wave

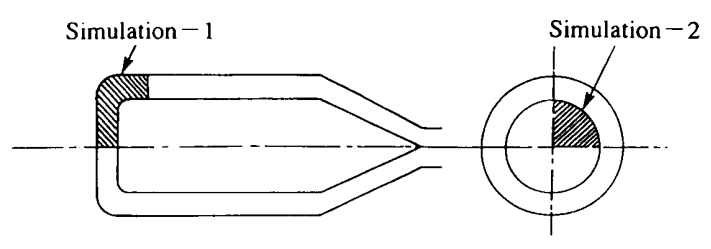

(a)

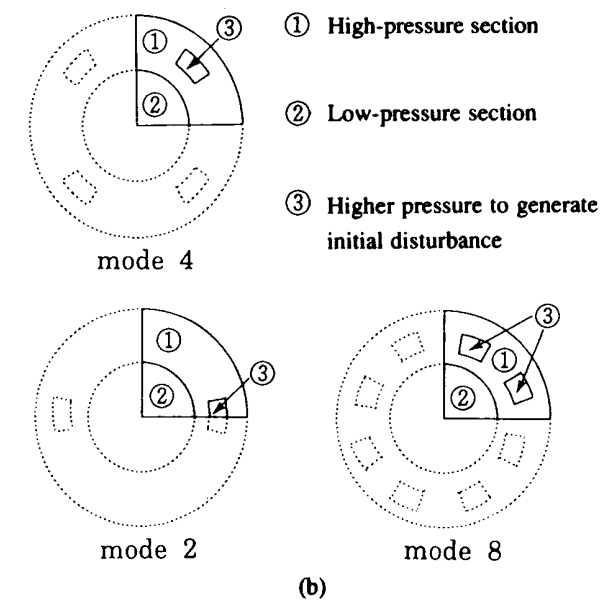

Fig. 2 Region of numerical simulation

\section{1 Computational Model}

3.1.1 Simulation 1 The computational region was the area around the axisymmetric $90^{\circ}$ bend and the convergence region. However, in this model, no initial disturbances were considered : the computation was intended to predict shock wave movement in the $90^{\circ}$ bend and convergence in the test section. The initial shock Mach number $\left(M_{s}\right)$ ranged from 1.1 to 2.0 .

3.1.2 Simulation 2 In this simulation, only the circular convergence region was considered. Initial disturbances were superimposed on the high-pressure section and their influence on shock convergence was examined.

3.1.3 Basic equations The conservation form of the Euler equations for inviscid compressible flow in Cartesian coordinates $(x, y)$ can be expressed in general curvilinear coordinates $(\xi, \eta)$ as,

$$
\frac{\partial \hat{U}}{\partial t}+\frac{\partial \hat{F}}{\partial \xi}+\frac{\partial \hat{G}}{\partial \eta}+\hat{W}=0
$$

where vector $\hat{U}$ represents the conserved quantities, $\hat{F}$ and $\hat{G}$ represent numerical fluxes, and $\hat{W}$ represents an inhomogeneous term in the axisymmetric flow.

$$
\left.\begin{array}{l}
\hat{U}=\frac{U}{J} \\
\hat{F}=\frac{1}{J}\left(\xi_{x} F+\xi_{y} G\right), \widehat{G}=\frac{1}{J}\left(\eta_{x} F+\eta_{y} G\right), \\
\hat{W}=\frac{W}{J}
\end{array}\right\}
$$

where $J$ is the Jacobian given as,

$$
\begin{array}{ll}
J=\xi_{x} \eta_{y}-\xi_{y} \eta_{x} & F=\left[\begin{array}{l}
\rho \\
\rho u \\
\rho v \\
\rho u^{2}+p \\
\rho u v \\
(e+p) u
\end{array}\right] \\
G=\left[\begin{array}{l}
\rho v \\
\rho v^{2}+p \\
(e+p) v
\end{array}\right] & W=\left[\begin{array}{l}
\rho u / x \\
\rho u^{2} / x \\
\rho u v / x \\
(e+p) u / x
\end{array}\right]
\end{array}
$$

where $p, \rho, u, v$ and $e$ are the pressure, density, $x^{-}$ component velocity, $y$-component velocity and total energy per unit volume, respectively. In simulation 2 , the inhomogeneous term $W$ in Eq. (4) was excluded from the basic equations. However, the simulation was conducted in cylindrical coordinates.

For a perfect gas the pressure is given by

$$
P=(\gamma-1)\left\{e-\frac{\rho}{2}\left(u^{2}+v^{2}\right)\right\},
$$

where $\gamma$ is the ratio of specific heats and is constant, since real gas effects are neglected.

\section{2 Initial conditions}

In simulation 1, the initial Mach number ranged from 1.1 to 2.0 to match the experimental conditions. 
The normal incident shock a short distance upstream of the $90^{\circ}$ bend was taken as the initial condition. A total of $351 \times 25$ grid points were used.

Simulation 2 is equivalent to a cylindrical imploding shock tube problem having high-pressure regions distributed around the outer boundary, as shown in Fig. 2(b). The computational domain was a quarter section of the circular flow field. The high-pressure section is assumed to be initially isothermal. The ratio of high pressure to the initial low pressure was assumed to be 10 . The initial disturbance was specified by excess pressure variations, which were expected to simulate the influence of disturbances caused by the struts. However, the present numerical model is idealized in the sense that an initially planar shock cannot be generated in the annular tube ahead of the struts because there is no ring-shaped diaphragm which can be ruptured simultaneously in space and time. Nevertheless, this model was useful for interpreting the onset of instabilities. The numerical simulation was carried out for initial excess pressures of $10 \%, 50 \%$ and $200 \%$, respectively. Mode numbers, $m=2,4$ and 8 , as in Fig. 2( b ) were studied. A total of $401 \times 151$ grid points were used.

\section{Experimental Setup}

\section{1 Shock tube and test section}

A $50 \mathrm{~mm}$ inner diameter (i.d.) shock tube was used to produce planar shock waves. This was connected to a coaxial annular shock tube which consisted of two coaxial tubes having an inner and outer diameter of $210 \mathrm{~mm}$ and $230 \mathrm{~mm}$, respectively, as shown in Fig. 3. The inner tube was supported by two sets of four equiangularly distributed struts from the outer tube, and its upstream conical section had an apex angle of $45^{\circ}$. The struts consisted of $18 \mathrm{~mm}$ outer diameter (o.d.) cylinders. The clearance between the inner and outer tubes was adjusted to be $10.0 \pm 0.02$ $\mathrm{mm}$. The blockage ratio of the struts to the annular shock tube cross section was 0.12 . The coaxial annular section was $500 \mathrm{~mm}$ long. It was expected that a

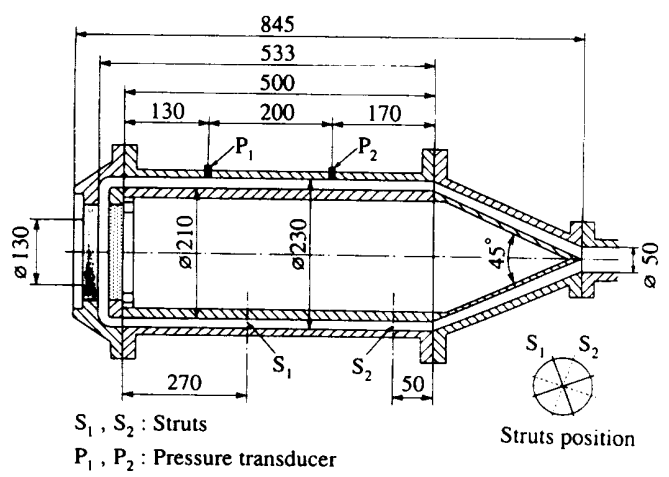

Fig. 3 Annular shock tube long straight coaxial section was preferable to attenuate the non-uniformities of the shock wave caused by the struts and cone tip. As was found, the longer coaxial section used in the present study, proved to be more effective in attenuating the shock wave than those used in previous works ${ }^{(3),(4)}$.

An axisymmetric $90^{\circ}$ bend was connected to the end of the co-axial annular section to transform the shock wave into a stabilized converging cylindrical shock wave ${ }^{(5)}$. Configurations of the bend significantly affected the movement and instability of converging cylindrical shock waves near the center of convergence. In our previous preparatory experiments ${ }^{(12)}$ on shock propagation over two-dimensional $90^{\circ}$ bends, we found that an optimal $90^{\circ}$ configuration existed, which smoothly transformed the shock waves and was a smoothly converging and diverging shape in the transformation. It was also found that a smoothly curved bend was preferable to a sharp $90^{\circ}$ bend. In the present series of experiments, such a simple, smoothly curved bend was used, and the radial gap between the outer and inner corners of the bend was maintained at $10 \mathrm{~mm}$. The radii of the inner and outer corners of the bend were $2.5 \mathrm{~mm}$ and $12.5 \mathrm{~mm}$, respectively.

A $130 \mathrm{~mm}$ o.d. glass observation window was installed in the outside wall of the test section. A 130 $\mathrm{mm}$ o.d. aluminum-plated glass mirror was placed on the inside wall, with the coated surface in contact with, and facing the flow. The gap between the mirror and window was $10 \mathrm{~mm}$. For the experiments, the test gas was air, at initial pressures that ranged from 5 $\mathrm{kPa}$ to $100 \mathrm{kPa}$. In order to measure the incident shock Mach number, two pressure transducers (Kistler $603 \mathrm{~B}$ ) were placed on the outer annular section at a $200 \mathrm{~mm}$ interval. The time differences between these pressure signals were measured with a digital counter and then average shock Mach numbers were calculated. Incident shock Mach numbers $\left(M_{s}\right)$ ranged from 1.1 to 2.0 in air.

\subsection{Holographic interferometry}

Figure 4 shows a schematic diagram of the holo graphic interferometric system, which is similar to the conventional shadowgraph method, except for the addition of a reference beam. The optical system, which is very similar to Twyman-Green interferometry ${ }^{(13)}$, consisted of two paraboloidal schlieren mirrors, two beam splitters, mirrors and lenses. By combining different image-focusing lenses, the magnification of the image of the test section on the film could be arbitrarily changed and a desired value chosen. In the present experiment, the magnification was approximately 0.5 .

A holographic double-pulse ruby laser (Apollo Lasers Inc. 22DH, $25 \mathrm{~ns}$ pulse duration and $2 \mathrm{~J} /$ pulse) 
was the light source. The ratio of light intensities of the reference and object beams was adjusted to range from $2: 1$ to $3: 1$, using neutral density filters or by

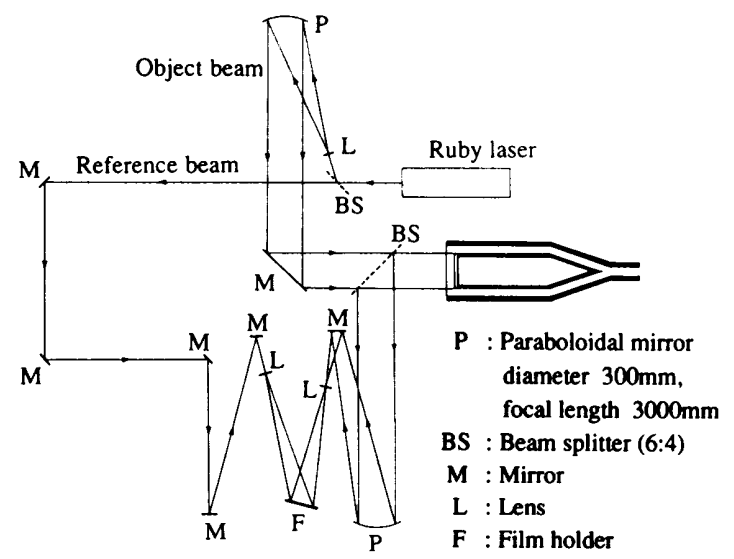

Fig. 4 A schematic diagram of the holographic interferometric system

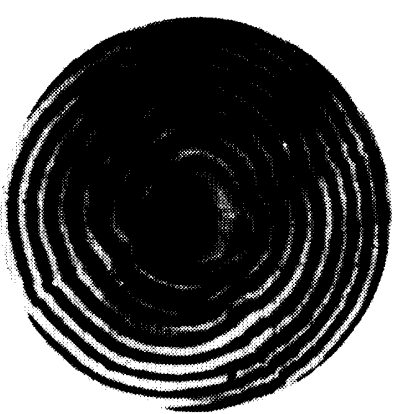

(a) $t=1135 \mu \mathrm{s}$

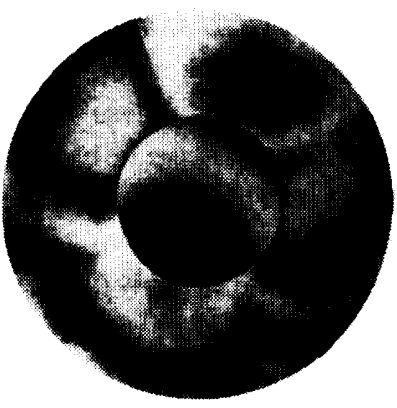

(e) $t=840 \mu \mathrm{s}$

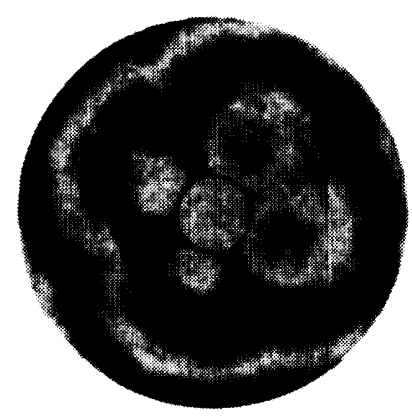

(i) $t=638 \mu \mathrm{s}$

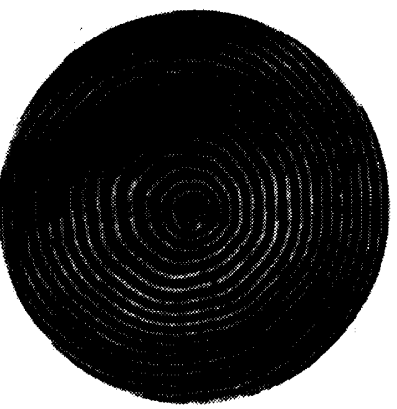

(b) $t=1161 \mu \mathrm{s}$

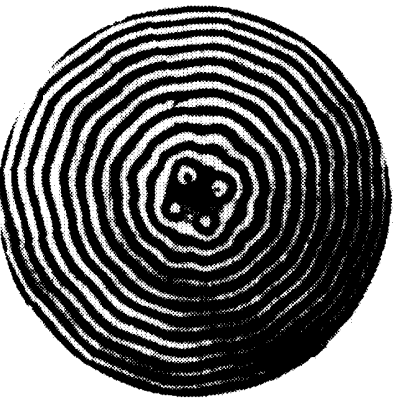

(f) $t=856 \mu \mathrm{s}$

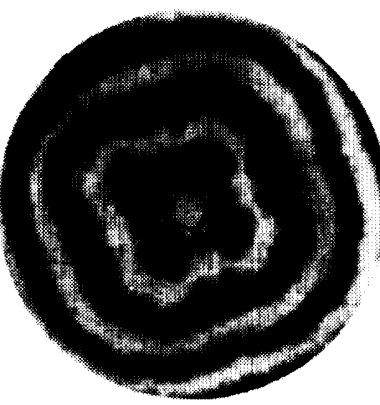

(j) $t=643 \mu \mathrm{s}$

changing the angle of the diverging object beam through the beam splitter to the paraboloidal mirror. The angle between the object and reference beams was approximately $20^{\circ}$ and the path difference between these beams was adjusted to be less than 50 $\mathrm{mm}$. The holographic film was $100 \mathrm{~mm} \times 125 \mathrm{~mm}$ sheet film, AGFA 10E75. For reconstruction, an argon-ion laser ( $514.5 \mathrm{~nm}$ wavelength and $1 \mathrm{~W}$ ) was used.

\section{Results and Discussion}

\section{1 Interferograms}

Sequential interferograms are shown in Fig. 5. For each Mach number, several experiments were repeated with identical initial conditions. The repeatability of the incident shock Mach number was maintained within $2.5 \%$ for each nominal incident Mach number. Figures $5(\mathrm{a})-(\mathrm{d}), 5(\mathrm{e})-(\mathrm{h})$ and $5(\mathrm{i})-(\mathrm{m})$ show reconstructed infinite fringe interfer-

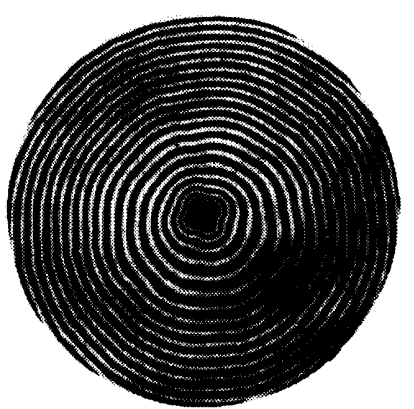

(c) $t=1167 \mu \mathrm{s}$

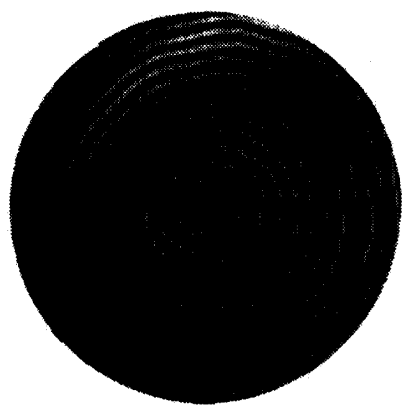

(g) $t=862 \mu \mathrm{s}$

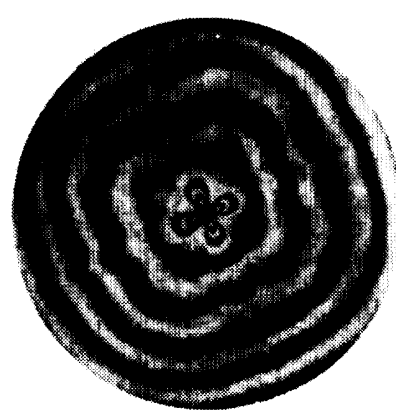

(k) $t=645 \mu \mathrm{s}$

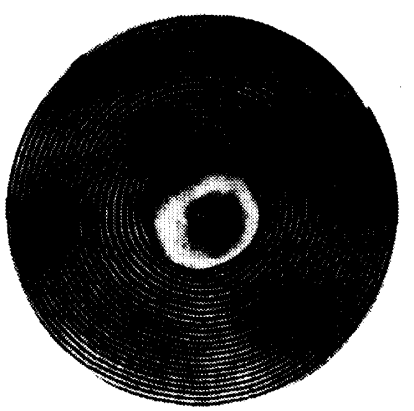

(d) $t=1208 \mu \mathrm{s}$

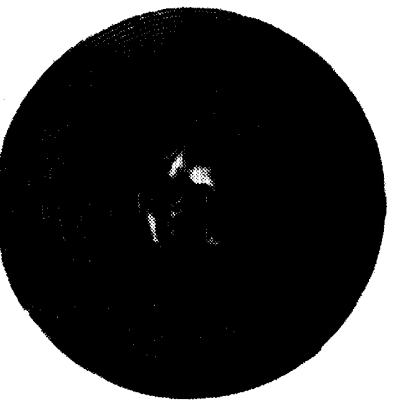

(h) $t=882 \mu \mathrm{s}$

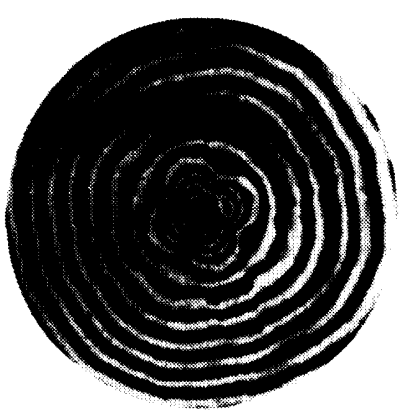

(I) $t=650 \mu \mathrm{s}$

Fig. 5 (a) $-(1)$ : Interferograms, (a ) $-(\mathrm{d}): M_{s}=1.1$ (e) $-(\mathrm{h}): M_{s}=1.5$, ( i ) $-(1): M_{s}=2.0$ 
ograms for $M_{s}=1.1,1.5$ and 2.0 , respectively. The displayed elapsed time started when the transmitted shock wave passed the first pressure gauge, as seen in Fig. 3.

5.1.1 $\boldsymbol{M}_{\mathrm{s}}=\mathbf{1 . 1}$ In Fig. 5( a ), a $16 \mathrm{~mm}$ diameter cylindrical shock wave can be seen converging towards the center. Although the shock wave appears to be cylindrical, a slight noncylindrical uniformity can be observed behind the shock wave in the fringe distribution. The fringe distribution was disturbed in a quadrangular manner which indicated the onset of mode-four instability. In Fig. 5 ( b ), the shock wave is no longer cylindrical but deformed, and the flow nonuniformity behind the shock wave is even more pronounced. The fringes near the shock resemble a four-leaf clover. One fringe shift corresponds to a density change of about $12 \%$ of the standard condition. In Fig. 5(c), the shock wave has propagated towards the center and the four-leaf clover shape has become much clearer, showing that the density nonuniformity has been enhanced. It was found that the position of these clover leaves was directed to the radial position of the four struts located in section $S_{1}$ in Fig. 3.

Thus, the initially small effect of the disturbances caused by the struts on the shock wave and the flow field behind it, as seen in Fig. 5(a), was amplified as the shock wave converged to the center in Fig. 5(c). Figure 5(d) shows the reflected shock wave, which becomes cylindrical with propagation outward and is known to be stable. Behind the reflected shock wave, pressure and density are nearly uniform; few fringes can be seen. Near the convergence center a small cross shape can be seen. This is, as is explained later, due to the remaining vortices. Each vortex is produced by a three-shock confluence, which provides evidence that Mach reflection occurred near the center. Perry and Kantrowit $z^{(3)}$ visualized shock convergence by shadowgraph and concluded from their photographs that weak shock convergence was stable. However, as shown in Figs. 5(c) and (d), the converging shock wave could not be cylindrical because vortices remained behind the reflected shock wave. This indicates that the converging shock wave was not stable even for $M_{s}=1.1$.

5.1.2 $M_{s}=1.5 \quad$ In Fig. 5( e ), at an early stage of convergence, the shock wave is nearly cylindrical with a $27 \mathrm{~mm}$ diameter. Although in Figs. 5( e ) - ( h ), similar to Figs. 5(a)-(d), the converging shock wave was initially nearly cylindrical, it became, with subsequent convergence towards the center, more significantly disturbed. Again, the form was modefour instability. The shock wave very close to the center appeared to be square, as seen in Fig. 5( $\mathrm{f}$ ).
Being initially negligibly small, the shock deformation grew larger with inward propagation.

However, the shock deformation cannot grow infinitely large and still be continuous. Large and continuous shock deformation induces large and continuous changes in flow variables along the shock front. However, this cannot be sustained if the gradient of flow variables becomes very large along the shock front. Such large shock deformation, finally, cannot be continuous and the transition to Mach reflection occurs. In Fig. 5( g ), larger shock deformation has terminated and the triple points having a three-shock confluence, composed of an incident shock, a reflected shock, a Mach stem and a slip line, are formed. This tendency was even more significant for stronger shock waves, as seen, for example at $M_{s}$ $=2.0$.

Figure 5(h) shows the shock wave from reflected the center. When transition from mode-four instability to Mach reflection occurred, four pairs of triple points were created, followed by four pairs of slip lines or vortices. These vortices collided mutually at the center where they remained, since the particle velocity behind the reflected shock wave was so small.

5.1.3 $M_{s}=\mathbf{2 . 0}$ Figure 5 (i) shows a cylindrical shock wave with a mean diameter of approximately $12 \mathrm{~mm}$, already slightly disturbed, as it converges towards the center. For this stronger shock wave case, the shock wave became noncylindrical at a much earlier stage. The noncylindrical deformation of the shock wave was again attributable to shock interaction with the struts, which supported the inner coaxial annular tube. In weaker shock waves, these disturbances seemed to be well suppressed, especially at the earlier stage, but in the case of the stronger shock wave, they always remained in the shock wave. In Fig. $5(\mathrm{j})$, the deformations had become Mach reflections. The three-shock confluences, i.e., the converging cylindrical shock as an incident shock, reflected shocks, straight Mach stems and slip lines, are clearly seen. Since in previous experimental work, the shadow or schlieren methods were mainly used, the nonuniformity of flow behind the converging cylindrical shock wave was not easily detectable. It should be noted again that the present holographic interferometer was particularly useful in detecting the earliest onset of instability in the flow preceding deformation of the shock wave. In Fig. 5(k), once Mach reflections had appeared, the triple point was selfpreserved. These three-shock confluences were predictable by solving the Rankine-Hugoniot relation for oblique shock waves. Figure $5(\mathrm{k})$ shows typical mode -four instability. In Fig. 5(1), the reflected shock wave diverged from the center and was again stable 
even for strong shock waves.

\subsection{Influence of the bend}

Figure 6 shows, for simulation 1, the numerical result of shock Mach number variation along the outer and inner walls and along the center line of the $90^{\circ}$ bend for $M_{s}=2.0$. In Fig. 6 , the ordinate is shock Mach number and the abscissa is a nondimensional distance along the bend, the origin 0 corresponding to the upstream uniform condition ahead of the bend and the convergence center being designated as 1 . Along

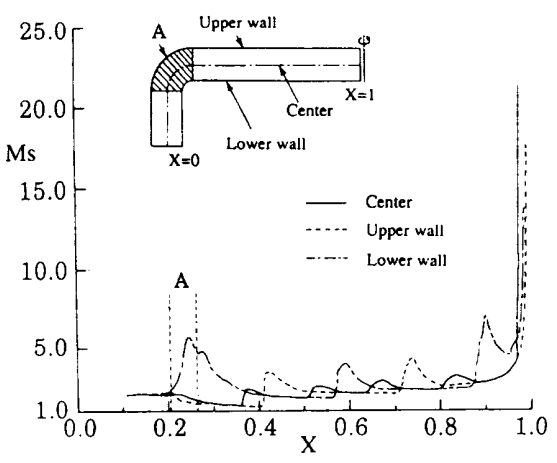

Fig. 6 Shock Mach number variation

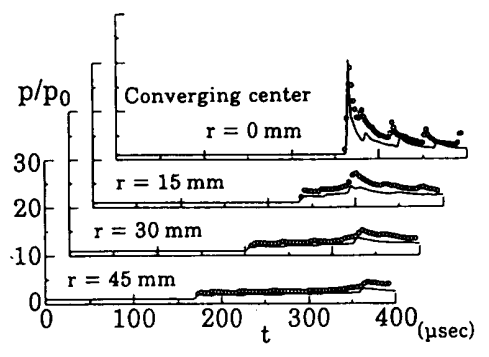

(a)

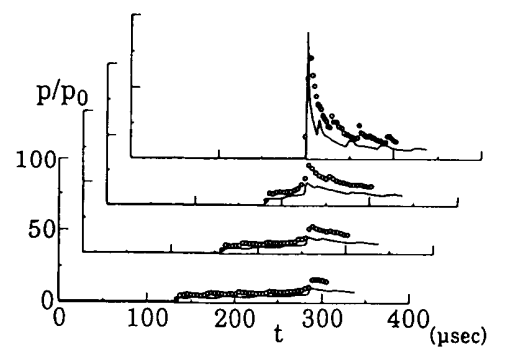

(b)

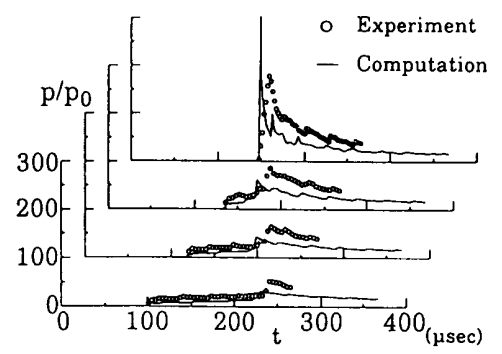

(c)

Fig. 7 (a) $-\left(\right.$ c ) : Pressure measurements, (a ): $M_{s}=1.1$, (b) : $M_{s}=1.5$, (c) $: M_{s}=2.0$ the outer and inner walls and along the center line, the shock Mach number increased with shock convergence. However, the shock Mach number did not vary monotonically: maxima existed during the convergence process, indicating that the shock wave was repeatedly locally accelerated and decelerated. This was due to the fact that, after the incident shock wave passed through the $90^{\circ}$ bend, the transmitted shock wave was reflected and diffracted between the upper and lower walls of the test section. The triple point on the transmitted shock, the so-called shock-shock, moved along the shock wave and was reflected repeatedly between upper and lower walls. Therefore, the slip lines remaining in the test section formed a zigzag pattern. This trend has been clearly observed in previous two-dimensional shock tube experiments. The shock Mach number distributions along the outer and inner walls show several peaks.

In the present holographic flow visualization, the converging shock wave was showed observed to have plausible thickness. This is attributable to the fact that the triple point exists on the converging shock wave and consequently the converging shock is curved. Therefore, when collimated light passed through the test section parallel to the shock wave, the deformed shock was observed to have thickened. This indicated that in using the annular shock tube, the cylindrical shock convergence process may be slightly three-dimensional.

\section{3 Pressure distribution}

Figures 7(a), (b) and (c) show pressure measurements for $M_{s}=1.1,1.5$ and 2.0, respectively. Pressure was measured $15 \mathrm{~mm}, 30 \mathrm{~mm}$ and $45 \mathrm{~mm}$ from the center. Pressure transducers (Kistler 603B) were equipped with a $2.5 \mathrm{~mm}$ diameter pressure-sensitive area and were placed on the outer wall of the test section. Figure 7 also compares the experimental results with the numerical simulations. Computational pressure histories at these points were evaluated by integrating computational pressures over several grid points, which corresponded to an area equivalent to the sensitive area of the pressure transducers. Good agreement was obtained between the experimental and computational values. However, the computed maximum pressures differ marginally from the experimental results, since initial disturbances were neglected and viscosity was not taken into account in the present computation.

\section{4 Numerical simulation with initial distur- bances}

Figure 8 shows the isopycnics for $M_{s}=2.0$ obtained in simulation 2 with an initial excess pressure of $100 \%$. In the experiments, the positions of the triple points were maintained in an angular sense. In 
the numerical simulation in Figs. 8(b) and (c), triple points collided mutually and reflected as if they were moving in a circumferential direction. The isopycnics, which resembled four-leaf clovers also appeared to be rotating. Strict quantitative comparison of the present numerical results with the experimental results in Fig. 5 is not possible simply because there are some differences in initial conditions between the experiment and the simulation. However, it is clear that each initial disturbance is amplified with shock convergence and is finally transformed into a Mach reflection.

Figure 9 shows the pressure distribution along the shock front for various initial disturbances, i.e., initial excess pressures. The ordinate is the pressure ratio

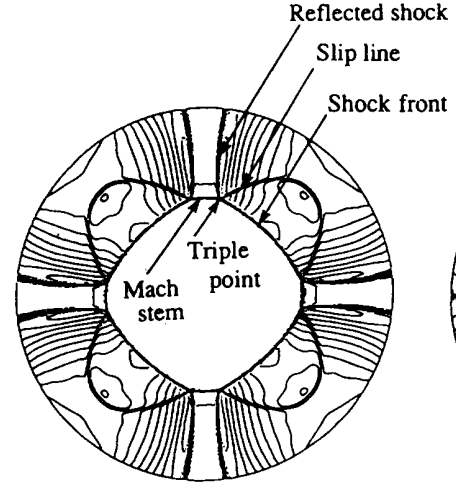

(a)

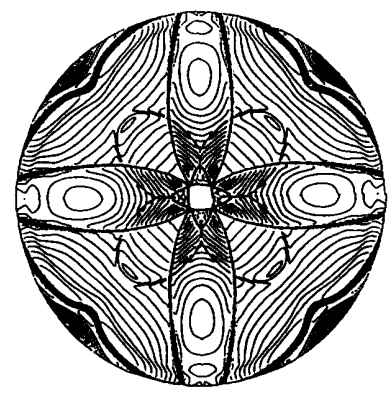

(c)

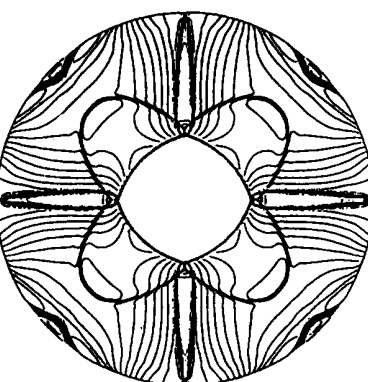

(b)

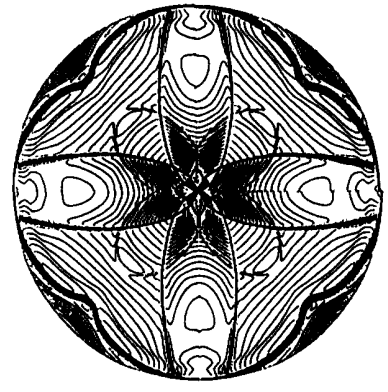

(d)
Fig. 8 (a) $-(\mathrm{d})$ : Isopycnics, $\operatorname{mode}=4$, ( a ): $t=56 \mu \mathrm{s}$ (b) : $t=63 \mu \mathrm{s}$ (c): $t=70 \mu \mathrm{s}$ (d): $t=72 \mu \mathrm{s}$ based on the initial pressure and the abscissa is the circumferential angle. In Fig. 9(a), the converging shock waves were not significantly disturbed but the disturbance-induced pressure was gradually amplified with propagation. Only in the case where $t_{5}=72 \mu \mathrm{s}$ for the reflected shock, did a substantial nonuniformity emerge.

In Fig. 9( b ), with an excess pressure of $50 \%$, the initial disturbance propagates circumferentially and is amplified. The broken line in Fig. 9(b) shows a trajectory of the nonuniformity which moves laterally until it is reflected and accompanied by distinct pressure jumps. This indicates that Mach reflection has occurred. It is found that high overpressure appears at the center of convergence. This trend was also seen in Fig. 6 at the shock convergence center. Comparatively uniform pressure appeared behind the reflected shock, which agreed well with the experiment, since in the experiment only a few fringe shifts existed behind the reflected shock.

In Fig. 9(c), for the case of $200 \%$ excess pressure, a drastically sharp pressure increase can be seen with the corresponding preceding formation of Mach reflection, as observed in Figs. $5(\mathrm{i})-(1)$. It was found that as the size of the initial disturbance increase, the peak pressure ratio also increase. For a given initial shock Mach number with a small initial disturbance, the average pressure along the shock front was increased and its distribution became comparatively uniform. However, when the initial disturbance was larger, the average pressure along the shock wave was not as high as in the weak initial disturbance case, but the local peak pressure around the shock front at the center was increased.

Figures 10(a) and (b) show the isopycnics for mode number $m=2$ and 8 , respectively. Initial excess pressure disturbance is $100 \%$. It is clear that the number of triple points corresponds to the number of initial disturbances. By increasing the mode number, shock interaction near the convergence center

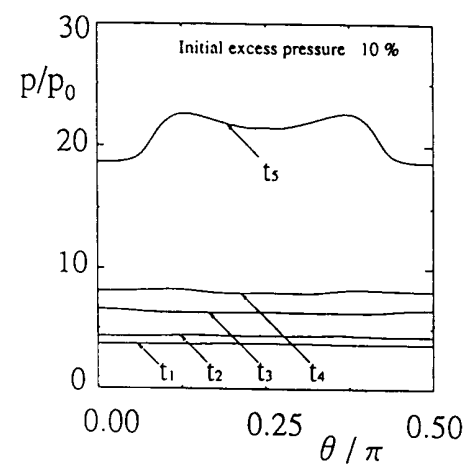

(a)

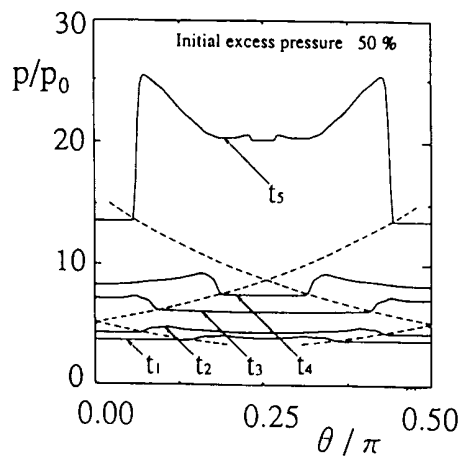

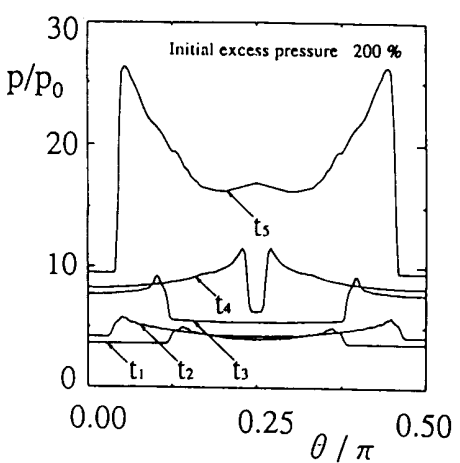

(c)

Fig. 9 (a) $-(\mathrm{c})$ : Pressure distribution, $t_{1}=44 \mu \mathrm{s}, t_{2}=56 \mu \mathrm{s}, t_{3}=68 \mu \mathrm{s}, t_{4}=70 \mu \mathrm{s}, t_{5}=72 \mu \mathrm{s}$ 
becomes more complicated.

\subsection{Shock Wave Distortion}

5.5.1 Measurement from interferograms In ideal cylindrical shock convergence, the cylindrical shape would always be maintained and the final shock convergence at the center would produce high pressure and temperature. However, it is not easy to

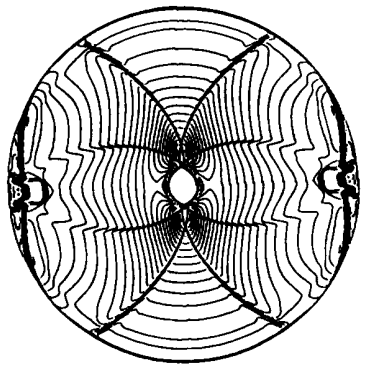

(a)

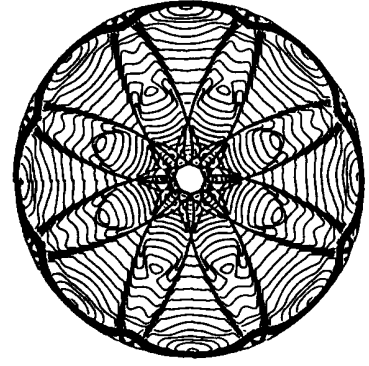

(b)
Fig. 10 (a )-(b): Isopycnics, (a ): $m=2,($ b ) : $m=8$ achieve this condition physically. In the present series of experiments, we have seen that the initial disturbance of the shock wave, however small, was gradually amplified with convergence. Figures $11(\mathrm{a})-(\mathrm{c})$, (d) - ( f ) and ( $\mathrm{g}$ ) - (i ) show distortions, from the cylindrical shape, of the converging shock waves, which were evaluated from the interferograms shown in Fig. 5, for $M_{s}=1.1,1.5$ and 2.0, respectively. The ordinate is the radius of the converging shock normalized with the mean radius. The abscissa is the circumferential angle $\theta / 2 \pi$. The deviation of the radii was always found to have four coherent peaks, due to the growth of mode-four instabilities associated with the struts. Small initial disturbances caused by the struts were transferred to the test section and, at an early stage of convergence, deformed the cylindrical shock wave shape very slightly. This instability grew with shock convergence. For $M_{s}=1.1$, the initial deformation was so small that at the initial stage the shock wave was cylindrical, as shown in Fig. 9(a). How-

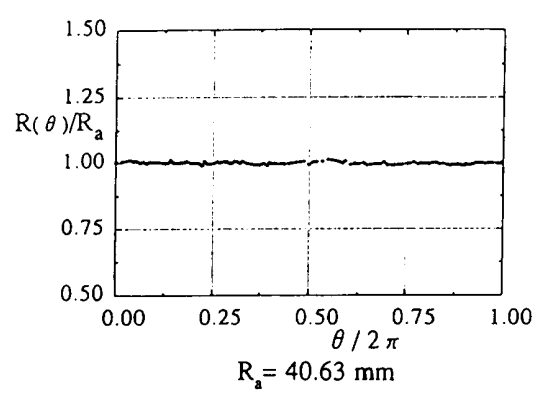

(a)

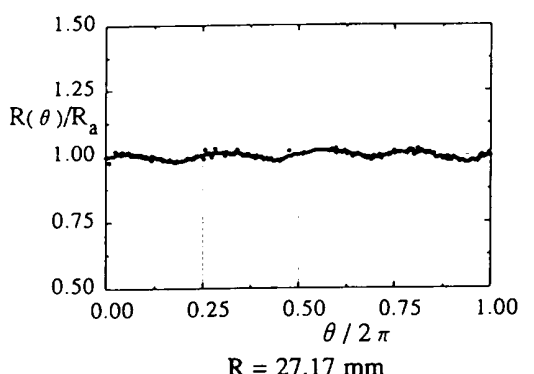

(d)

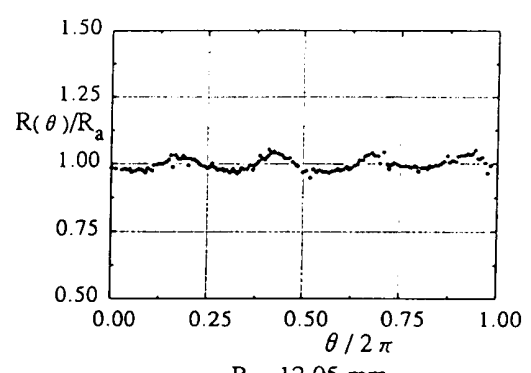

$\mathrm{R}_{\mathrm{a}}=12.05 \mathrm{~mm}$

(g)

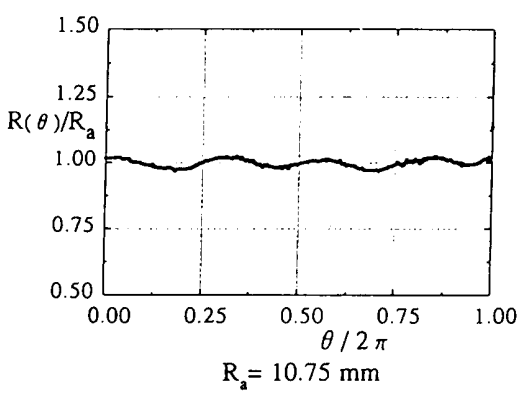

(b)

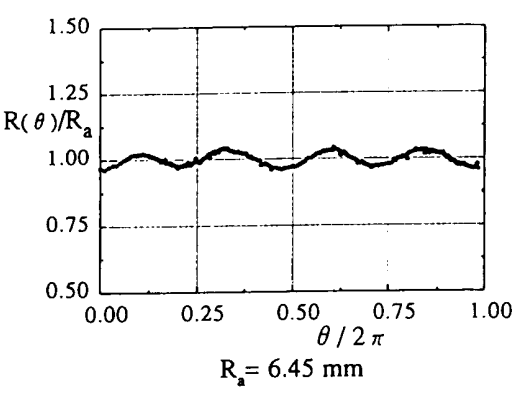

(e)

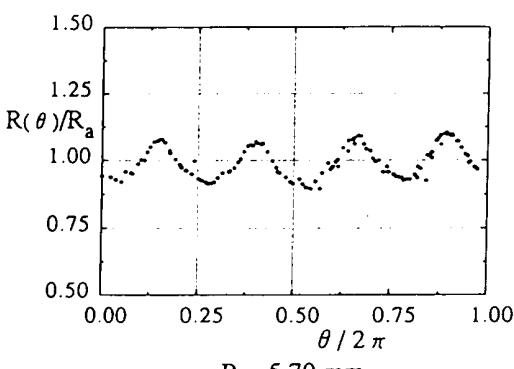

$\mathrm{R}_{\mathrm{a}}=5.70 \mathrm{~mm}$

(h)

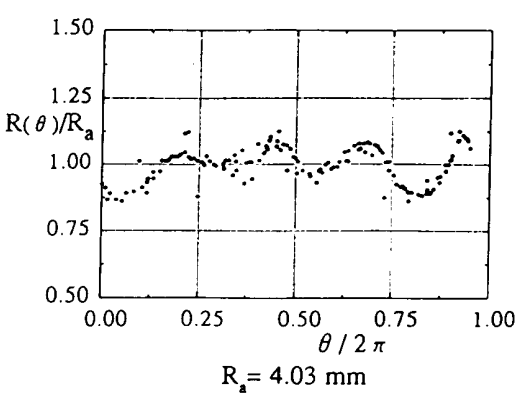

(c)

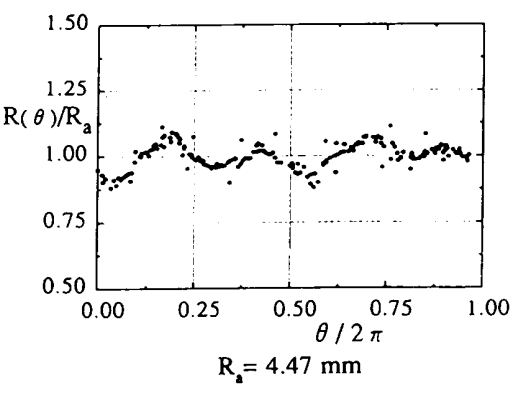

(f)

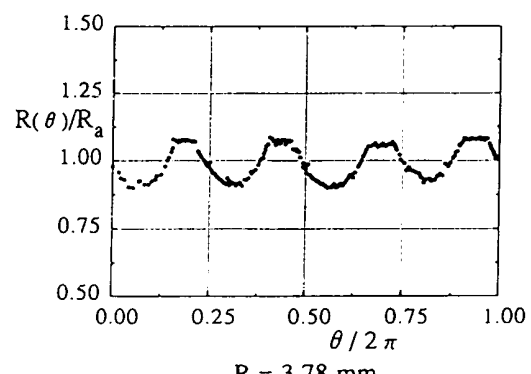

(i)

Fig. 11 (a) -( i ): Distortion of the converging cylindrical shock wave, (a)-(c): $M_{s}=1.1$, (d)-( f): $M_{s}=1.5$, (g) $-($ i $): M_{s}=2.0$ 
ever, shock deformation grew later to a noticeable level, as shown in Fig. 11(c).

For $M_{s}=1.5$, this trend was more pronounced. The initial disturbances already present at $R_{a}=27$ mm were gradually amplified, as shown Figs. 11(d)(f ). A typical example for $M_{s}=2.0$ is given in Figs. $11(\mathrm{~g})-(\mathrm{i})$. Small deformations grew to the extent that they could not be accounted for by small perturbation theory. The increasingly large deformation of the converging shock wave finally led to the formation of Mach reflection at each distortion site on the shock wave. In Fig. 11 ( $i$ ), the flat topped shock deformation clearly indicated discontinuities caused by Mach reflection, as seen in the corresponding interferogram, Fig. 5( $j$ ). It is concluded that the converging cylindrical shock wave is unstable even for weak shock waves, and the initial small deformation grows with shock convergence, resulting in the deterministic nonlinear behavior of triple-point formation. The present sequential observation of shock deformation can be used to obtain direct time and spatial variations of flow properties behind the converging shock.

5.5. 2 Amplification Figure 12 summarizes the amplification of shock wave distortion. The experimental results were taken from Fig. 11. The ordinate

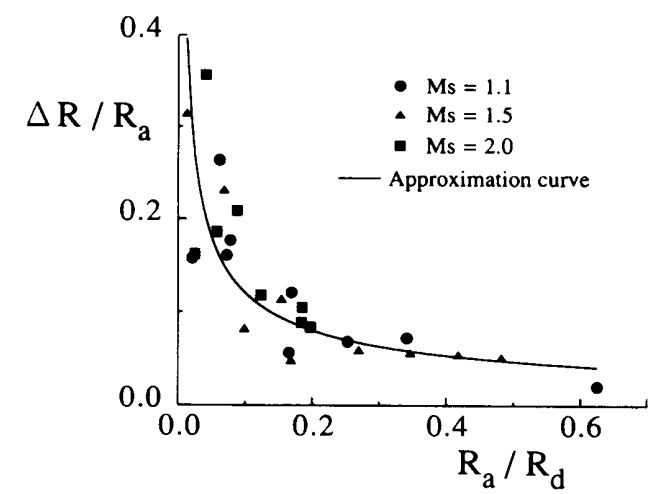

Fig. 12 Amplification of the shock front distortion in experiments

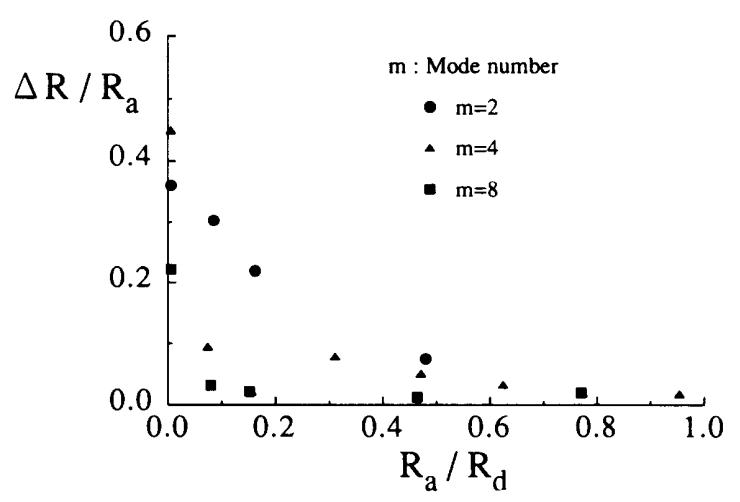

Fig. 13 Numerical simulation of amplification of the shock front distortion with an excess pressure of $100 \%$ is $\Delta R / R_{a}$, deviation of the shock wave $\Delta R$ normalized with the mean radius of the converging shock $R_{a}$, and the abscissa is the mean radius $R_{a}$ normalized with the characteristic length, which is the radius of the observed section $R_{d}=65 \mathrm{~mm}$. In Fig. 12 small shock deformation increased rapidly when the shock wave was inside the range $R_{a} / R_{d}>0.2$. This steep rise in deviation of the radius is related to the transformation by the influence of small perturbations to the formation of a triple point. Finally, it is found that the dimensionless shock deformation seems to be independent of the incident Mach number. The solid line represents the fit of an empirical curve.

The effect of mode numbers 2,4 and 8 on the amplification of shock deformation is compared in Fig. 13. $\Delta R / R_{a}$ is plotted against the mean radius $R_{a} / R_{d}$. It is readily seen that, inside the range $R_{a} / R_{d}<$ 0.5 , there is a trend towards increasing shock deformation with decreasing mode number. However, the trend is inconsistent between modes 2 and 4 at $R_{a} / R_{d}$ $=0.01$, at a corresponding shock radius of $0.65 \mathrm{~mm}$. This occurs at the limit of the resolution of the computation, which is therefore less accurate. In the present computation, initial excess pressure for all the initial disturbances for mode numbers 2,4 and 8 was $100 \%$. Therefore, as seen in Fig. 13, if various mode numbers having unequal initial disturbances were present, a smaller mode number and larger initial disturbance would contribute to the final disturbance more strong. ly than any other combination of mode number and initial disturbance.

\section{Conclusions}

The results obtained are summarized as follows:

(1) Holographic interferometry is particularly useful for observing the early onset of instability in the flow field of a collapsing cylindrical shock wave preceding its transformation from a single wave to a deformed multiple wave.

(2) Initial small perturbations of the shock wave grew with shock propagation and, at the final stage, resulted in the formation of triple points.

(3) Following convergence, the flow nonuniformity behind the shock wave showed the onset of mode -four instability, which was due to the struts supporting the inner tube of the annular shock tube.

(4) The greater the severity of the initial disturbance and the smaller the mode number, the larger was the amplification of the shock wave distortion.

The authors would like to express their gratitude to Messrs. O. Onodera and H. Ojima of the Shock Wave Research Center of the Institute of Fluid Science, Tohoku University, for their assistance in conducting the present experiments. The authors also 
gratefully acknowledge Messrs. S. Hayasaka of the machine shop of the Institute of Fluid Science, Tohoku University, and K. Takahashi of the Shock Wave Research Center of the Institute of Fluid Science, Tohoku University, for manufacturing the shock tube.

\section{References}

(1) Takayama, K., Proceedings of international workshop on shock wave focusing, Shock Wave Research Center, Institute of Fluid Science, Tohoku Univ. (1990)

(2) Guderley, G., Starke kugelige und zylindrische Verdichtungsstösse in der Nahe des Kugelmittelpunktes bzw. der Zylinderachse, Luftfahrtforsch, 19 (1942), p. 302.

(3) Perry, R.W. and Kantrowitz, A., The production and stability of converging shock waves, J. Appl. Phys., Vol. 22 (1951), p. 878.

(4) Wu, J.H.T, Neemeh, R.A., Ostrowski, P.P. and Elabdin, M.N., Production of converging cylindrical shock waves by finite element conical contraction, In : Hertzberg, A. and Russell, D. (eds.) Proc. 11th Int. Symp. Shock Tubes and Waves, Washington Univ. Press, Seattle (1977), p. 107.

(5) Takayama, K., Kleine, H. and Grönig, H., An experimental investigation of the stability of converging cylindrical shock waves in air, Exp. Fluids, 5 (1987), p. 315.

(6) Gardner, J.H., Book, D.L. and Bernstein. I.B.,
Stability of imploding shocks in the CCW approximation, J. Fluid. Mech., Vol. 114 (1982), p. 41.

( 7 ) Itoh, T. and Abe, K., Simulation of instability of cylindrically converging shock waves, In : Proc 4th Int. Conf. Applied Numerical Modelling, Taiwan (1984), p. 666.

( 8 ) Demmig, F. and Hehmsoth, H.H., Model computation of converging cylindrical shock waves: Initial configurations, propagation, and reflection, In: Kim, Y.M. (ed) Proc. 17th Int. Symp. in Shock Waves and Shock Tubes (1989), p. 155.

(9) Watanabe, M., Stability of converging cylindrical shock wave, Master Thesis, Tohoku Univ. (in Japanese) (1989).

(10) Harten, A., High resolution scheme for hyperbolic conservation law, J. Comp. Phys., Vol. 49 (1983), p. 357.

(11) Itoh, K. and Takayama, K., Transonic shock tube flow over an NACA 0012 aerofoil and elliptical cylinders, In: Bershader, D. and Hanson, R.K. (eds) Proc. 16th Int. Symp. Shock Tubes and Waves, Stanford Univ. Stanford Press (1987), p. 693.

(12) Takayama, K., Honda, M. and Onodera, O., Shock propagation along 90 degree bends, Rept. Inst. High Speed Mech., Tohoku Univ., Vol. 35 (1977), p. 83.

(13) Takayama, K., Application of holographic interferometry to shock wave research, Proc. SPIE 398 (1983), p. 174. 